\title{
New Routing Paradigms for the Next Internet
}

\author{
Djamel H. Sadok, Luciana Pereira Oliveira, and Judith Kelner \\ Federal University of Pernambuco (UFPE), Brazil \\ $\{j a m e l, l p o, j k\} @ g p r t . u f p e . b r$
}

\begin{abstract}
This work suggests that current and future could be seen as flexible architecture structured by societies. Information sharing and routing are then studied in this context. A proposal, namely evidence-based knowledge sharing routing is described and evaluated. Information sharing in social networks looks to be a promising venue for next Internet routing.
\end{abstract}

Keywords: Social Networks, Information Sharing, Architecture, Internet and Networks.

\section{Introduction}

One may expect the next Internet to offer a new living space for working, entertaining, a sort of second life, rather than a simple transport or information distribution channel. Simply put, where there is information, there should be an Internet access to it. Despite the large debate on whether one should consider a clean slate approach for a "new" Internet, the authors chose to define a socially inspired flexible structure that may embrace many possible new networking contexts. For years we have been intrigued by the way bio-inspired models from ants, bees, humans and other animal species organize on a large scale their societies. The question is: how much would one gain by extending some of these models to the Internet.

A critical look shows that the current Internet already mimics human behavior to an extent, continuously spawning more and more virtual communities. Hence, elements of the next architecture(s) may take on roles characterizing them within their societies while using rules and policies to achieve their goals and make predictable decisions. Recent research from content centric networks [1] emphasized this. Here, routers should be seen as objects acting on content according to their context. Hence it is the context that defines the role and not otherwise.

In this paper, we propose and discuss the Internet as a structure based, among other things, on the concepts: 1) usage of a society-based structure with rules or policies governing their existence; 2) roles and relationships and finally; 3) knowledge sharing between society members for information routing. It is this last item, namely knowledge sharing, which is examined in this paper.

\section{Embracing the Societies for the Internet Architecture}

A simplified one size fits all approach is no longer practical. The authors see the next Internet as one that consists of different societies, governed by different policies, 
having different goals and technological characteristics. Somehow, these societies get together in an attempt to offer some common good or services.

Within a single society one expects to see relatively more homogeneity, stability, common rules and vocabulary (ontology) being applied. Some cultures become more dominant at some time of history, others remain peripheral to the global picture. Among societies, different views may be adopted to achieve similar objectives and to enforce internal autonomy. Despite diversity, societies need to talk to each other and harmonize with their environments. Hence one expects some order in such disorder, common, through probably weakly coupled, roles that ensure end to end cooperation.

The overlay networks are interesting model to exemplify societies. They constituted virtual communities like Peer-to-Peer (P2P) networks (such as Skype and BitTorent), Newgroups, mailing lists, video and music file sharing over physical network. They are exemplified by the context of global interworking in Fig. 1.

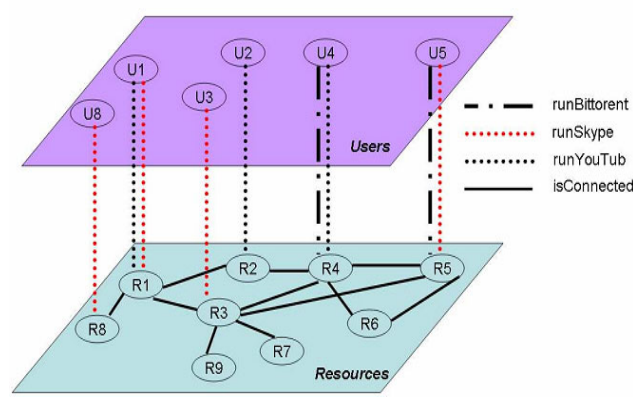

Fig. 1. View of a Society Based Internet

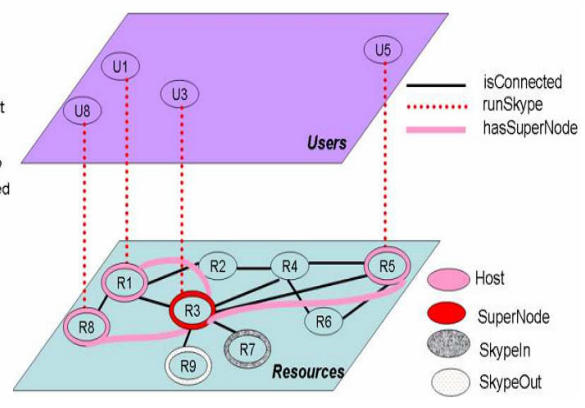

Fig. 2. Skype society

Fig. 2 depicts a Skype society extracted from the example in Fig. 1 [2]. For this scenario, connectivity resources include user desktops, routers and the public phone service (PSTN) gateways. The roles responsible for stability within this society include the following special servers: Supernodes, Skype-in and Skype-out gateways. A host can be a Skype client used to place voice calls and send text chat messages. A Supernode is a special one with a public address (or external role in society terminology) possibly having sufficient CPU, memory, and network bandwidth. Skype-in and Skype-out gateways reach users and applications from other communities or societies such as the public phone service and other VoIP application users. Hosts and Supernodes organize the P2P overlay, while the Skype-in and Skypeout elements provide PC-to-PSTN and PSTN-to-PC bridging.

We envisage a next Internet where, for example, Skype and Orkut users may talk to each other through special gateways. Similarly, YouTube users may get to know MSN and POTS users and interact with them. Future societies or groups would be plug-and-play connectible as long as they follow some simple proposed social structure and stability requirements.

Therefore, our societies must have some basic knowledge on how to forward such requests among themselves. A request could visit many societies in between, asking them about given knowledge or services, if they could have suggestions on forwarding a request. Such requests may die out slowly in the meta-societies 
hyper-space unless they locate their target society. Our approach implicitly adopts the late binding mechanism as users are not required to know the exact path or even have the full information on their communication partners. Time to live flags should be associated with forwarded queries or information for them to be removed when unsuccessful.

\section{Relevant Advances the Social Approach in Routing}

Many protocols have been based on social phenomena such as regional gossiping [3] and rumor routing [4]. The following are some new routing related concepts:

- Proximity: represents how nearby and further away a node is using a predefined distance concept;

- Knowledge: represents if someone knows, heard of, has never seen before or knows someone (make a referral to) who may help;

- Volatility: reflects the presence of highly dynamic societies with limited scope in time and space. A new emergency network setup to deal with an earthquake or one covering a sport event are such examples.

- Connectivity level: shows how high (important) a society is. It is therefore, likely, that we have different connectivity levels, including: global societies that are present all over the world and connect to many others; big, regular and small societies. A power Law or mass amount disparity phenomenon may be expected as many societies will have little connectivity and the global ones may be limited in numbers.

Data fusion strategies, such as the Dempster-Schaffer [5], [6] technique, may then be used to combine the evidence gathered by a node to make a routing decision. Such decisions may be cached for possible future reference. Although routing has been used to refer to mechanisms responsible for forwarding information, a more suitable name reflecting a new Internet scenario where societies are cooperating with each others could be knowledge sharing. Our scenario is that of a community of societies constantly conducting knowledge diffusion activities, by far, a wider scope.

Cultural diffusion models have not been effectively adopted for large scale systems such as the Internet. They have been developed to illustrate the processes of knowledge diffusion between knowledge workers, and factor the coefficients of distance, willingness, motivation, and ability of comprehension and expression [6].

Gossip-based models have, for instance, extensively been used in knowledge diffusion work [7]. This is however an oversimplified model, as we need to use asynchronous models where there is call for more interaction rather than relying on old acquaintances to lead with the future. In [8], a barter process is assumed between the members that can trade different types of knowledge.

Considerable work has also gone into partially connected networks [9], [10]and Epidemic Routing (ER) [11]. Such work introduces ER, where random pair-wise exchanges of messages among mobile hosts ensure eventual message delivery. Similarly, Chen and Murphy propose a protocol called Disconnected Transitive Communication which involves the application in locating the node among a cluster of currently connected nodes that it is best to forward the message to [12]. Given that 
messages are delivered probabilistically in ER, the application may require the use of acknowledgments. Some optimizations may be further made using techniques such as bloom filters [13].

Relevant ideas may also come from recent work on disruptive networks (DTN). Here a number of routing strategies have been evaluated including flooding, random walk, replica forwarding with staggered attempts [9], even enhanced link state protocols and hybrid approaches. Similarly, Zhang in [13] and Small and Haas in [14] studied analytically algorithms derived from ER. Spyropoulos proposed a multi-copy scheme for DTN routing in [15]. In [16], a DTN routing strategy that minimizes packet loss is developed.

\section{Knowledge Sharing and Routing}

The social routing algorithms are based on the observation that information reaches more people and destinations when going through popular nodes [17]. For such context, we suggest the knowledge network could be measured to classify the society's evolution and such classification could be coordinating the routing of messages.

\subsection{Evolution of Societies and Routing Information}

We expect that the first step in evolution of a society corresponds the processing where a society gets to know itself, who are its members, their reachability towards other societies and their capacity and willingness to work as gateways. We expect every society to implement some basic internal discovery mechanisms allowing it to reach one or more representative nodes, that know about its connections to other societies and has at least a partial description of what others do. The interaction among the societies will continue its evolution and consequently the classification changes.

Global societies will give priority to learning about each others. They would accept even weak evidence within passing routing information (or recent overheard gossip). The lower we go into the connectivity index, the less persuasive their evidence is considered. There could be an exception however, for example that of volatile (highly dynamic) societies. This class could be given special treatment as a slow spread of gossip may work against it due to the time it takes for other societies to know about it. Hence a society may inflate its connectivity or knowledge metrics just to ensure that the knowledge sharing converges rapidly allowing others to discover it. Alternatively, a society may chose to restrict its connectivity information and operate in an almost "silent mode". Note that this may also be subject to policy guidelines. We do not consider the effect of wrong or malicious information as part of this work at present. Apart from the global societies, each society seeks to know about similar and higher level ones. Although we already used the term "know about" yet we did not define this. So what are the implications of knowing about a given society? These are three: first its identification is no longer strange to your "gateways" or similar entities, its purpose (services) in life is known and third, we know some information on the societies it is capable of reaching i.e. its external topology. 
Unlike the existing Internet, duplicate knowledge is not removed but kept and some accumulative levels of evidence are associated to each of the entries. All evidence is sorted according to its levels and eventually the lower ones are removed. Even evidence may get old and consequently weaker to reflect the fact that old knowledge may no longer be valid. Special, per society policies should be defined to enforce such decisions. A lifetime may explicitly be associated with an evidence message by the issuing society.

Changes within the connectivity level of a society should be communicated to its siblings. When trying to find out about a path to a society, a query with a maximum society hop count may be made. Upon failure, this threshold may be increased. A lesson to be learned from this, is that knowledge of distant societies is more precious and should be maintained and spread to nearby siblings. A society that knows many distant societies could have its connectivity level increased (improving its status in a world of societies - where you are who you know!).

Unlike deterministic routing, societies may live with uncertainty and incomplete knowledge. We may simply need to locate a node that is close enough and avoid loops. By close we mean some society that knows about the destination. This is in line with current new Internet architecture proposals that use late binding. Nonetheless, we need to maintain delivery rates high while minimizing resource utilization. We also need to derive upper and lower bounds on the delay.

One or more metrics, both quantitative and even qualitative, need to be established to measure its effectiveness. As an example, we select a probabilistic metric called delivery predictability, $P(a \Rightarrow b) \in[0,1]$, at every node $a$ for each known destination $b$.

The routing could be seen as a multiple copy scheme that operates according to probability or predictability with support for both on-demands versus table driven modes. Routing needs to learn, which members lack some knowledge, and which member owns it. When knowledge is shared between two societies on a given destination or services, then a society's knowledge on that first one increases. Knowledge aging is also taken into account. Hence with time, it is expected that the delivery predictability to a given destination will decrease. This is similar to ant based routing that adopts the idea of dissipating pheromone trails.

Further, societies may be characterized by different coefficients of knowledge sharing willingness and knowledge learning motivation. It is also expected that a society detaining a high level knowledge may not be willing to share knowledge with one with a lower knowledge level. We need to characterize societies with wide ranging possible knowledge sharing willingness and knowledge learning motivation.

However, if there is a small gap between the knowledge of societies they probably reside within the same "class" of societies. A threshold representing the knowledge gap may be used to determine when societies should exchange knowledge. Distance between the members may also influence knowledge sharing. Such distance may be regarded as one of the following categories or some combination: 1) geographical distance; 2) cultural distance: such as languages, values, and 3) technology distance, etc;

\subsection{Elements of Proposal Routing Algorithm}

The proposed routing may be viewed as one that consists of the following algorithms or blocks: 
1. Knowledge sharing mechanisms: algorithms may be based on gossip, scoped broadcast, etc. These take into consideration knowledge sharing willingness of a given society, status of a society, local policies, etc.

2. Knowledge adoption algorithms: such class of algorithms determines when a society may accept some incoming information and how to associate a validity level to it. A receiving society maintains total autonomy in deciding whether it will accept new information. For example, it may determine that it is unwilling to carry messages larger than a given size or destined for certain societies. Hence we see room for policies in our proposal to govern the knowledge sharing and evidence acceptance in the future Internet. One may also assume an implicit trust model, where societies with the same degrees of connectivity (number of neighboring societies) trust the announcements made from others. We will not examine any further this point however in this work.

3. Society lookup: represents strategies that may be adopted in finding out the whereabouts of a given society and its reachability information. Such strategies may be based on intelligent algorithms, brute force, semantic queries, dedicated known overlay servers such as society Oracles, etc.

4. Strategies for the update and retrieval of society information: determine when and how to seek some information it owns and tell those interested of possible changes in its status. Examples of mechanisms that may be used to offer such functionalities range between publish and subscribe, push models, broadcast, etc.

5. Society clustering algorithms: may be seen as a future attractive mechanism for optimizing knowledge sharing. It may be modeled by averaging the proportion of neighbors of a society who are also connected together. Societies may also use third party knowledge to deal with forwarding issues. Such optimization steps are left for future studies.

\section{Knowledge Sharing Model}

In this work, we evaluate the first element of proposed routing algorithm (knowledge sharing) analyzing the society evolution. We modeled the algorithm such that its knowledge flow will be inversely proportion to the distance among societies, and directly proportion to knowledge gap. Existing Internet based social networks have been described as power-law or scale-free degree distribution networks with distinct epidemiologic properties from those of random networks [6]. We need to investigate how future Internet society will evolve and whether they would maintain a linear expansion of the existing power Law model or change into multiple different topologies. The network topology impact on knowledge diffusion models has been shown in [7]. 
Techniques such as adaptive percolation [18], [19] may be used to show that by propagating knowledge from a small number of specially chosen societies we can drastically increase the probability of the network becoming almost fully connected (aware of its societies and their whereabouts).

Attending such characteristics, our algorithm model was defined as $S=\left\{S_{i}: i \in N^{*}\right\}$ been a set of societies and \|S\| their cardinal. Let $d_{i}$ be the degree of the $i^{\text {th }}$ society $S_{i}$. We divide societies into $K$ classes $\left\{C_{l}, \ldots, C_{\mathrm{k}}\right\}$ according to their connectivity level or degree (of knowledge). This division is done in a way that it should take a long time for a society before being able to leapfrog to a next level in order to maintain a stable network connectivity state. Hence there should be a small number of classes that are distant enough. The simulated world (or the next Internet) starts up with a small number of classes and will grow to create new levels by continuously upgrading those with a larger degree of connectivity.

At the top level there are more stable societies with a high connectivity degree. A society gets first to know about its physically sibling neighbors through more than one interface or gateway. This will have a weight factor equal to one and reflects direct contact in this case. If a society learns about another one through a given path, it also knows about those along such path. Hence such knowledge is accumulative and may be represented by independent (separate) logical links within a graph for instance.

Let $l_{i, j}$ be a path to society $j$ from $i$ (information on society $j$ from $i$ ) and $\left\|l_{i, j}\right\|$ be the total number of such knowledge elements. Hence we have (1). Without loss of generality we may assume that a society $S_{i}$ with degree $d_{i}$ belongs to class $C_{k}$ with $k \in\{1 \ldots K\}$ given we have (2) and (3). Hence if we choose for example B as 100 and $\mathrm{K}$ as 4 then we have the four classes: $C_{1}, C_{2}, C_{3}$ and $C_{4}$ should have as members societies with degrees immediately bellow the thresholds $10^{2}, 10^{4}, 10^{6}$ and $10^{8}$. The larger the base $B$ is, the more distant are the classes. Hence we may view $\mathrm{B}$ as a tunable factor that separates the big from the riffraff. Let $N_{i}$ be the number of elements within a given class $C_{i}$ for $1 \leq i \leq K$.

$$
\begin{gathered}
d_{i}=\sum_{j=1 ; j \neq i}^{\|S\|}\left\|l_{i, j}\right\| \\
\mathrm{k}=\operatorname{Min}\left\{\mathrm{K}, \operatorname{Max}\left\{1, \text { Ceiling }\left(\log _{B} d_{i}\right)\right\}\right\} \\
C_{j}=\left\{S_{i}: B^{j-1} \leq d_{i}<B^{j}\right\} .
\end{gathered}
$$

In contrast to the static probability percolation strategy our approach uses a dynamic probability for responding a knowledge request from society $i$ about $j$ to its known colleagues given by (4) where $\alpha_{k}$ is a per class tunable constant. For our evaluations we assume that death may occur at any $C_{i}$ level according $p_{i}=b_{k} / d_{i}$, where $b_{k}$ is also a per class tunable constant. In other words, we can introduce different levels of death at the classes or define a limitation such as setting up societies at the bottom level of the stability graph $\left(C_{l}\right.$ class) to it only cease to exist. Knowledge sharing works by associating a weight for each new information. Knowledge related to a small society (limited connectivity degree) will have a smaller probability for being spread to the 
rest of the world. Hence it is very likely that only nearby societies know about a low degree one as this knowledge dies out the further we get from this society.

$$
p_{i, j} \sim \alpha_{k} * d_{i} * d_{j}
$$

Knowledge is exchanged under different conditions:

1. When two societies need to exchange knowledge. For example, when a new society is born, it is then eager to tell the world about itself;

2. A request is made to locate or search for a given society using some information known about it.

3. Currently, knowledge accumulates. However, we some knowledge may become unavailable when a society dies out. Others may be alerted about this;

\section{Evaluation of Knowledge Sharing Model}

The simulation studies the mechanisms to build paths that corresponds the evolution of the societies in terms of learning, accumulating and disseminating information (paths).

\subsection{Simulation Metrics and Parameters}

We evaluate the membership of each class. The presence of a large number of nodes in some class shows that its nodes learn more each round and more frequently. The dissemination of knowledge is a second potential metric to evaluate the knowledge flow. Societies propagate more response messages when the nodes are "young" as need to know more about their neighbors. The forwarding of lookup requests adds latency and increases the lookup traffic load. Hence, the analysis investigates both the accumulated knowledge as well as the frequency of learning and dissemination of knowledge. We assume that the network starts with a small number of nodes (set to 10 nodes here) and we simplify the simulation by using fixed numbers of classes and set the constant B to, respectively 4 and 10 . To reduce memory allocation requirements, each node may obtain up to a maximum of a hundred direct connections.

The following tunable $\alpha_{k}$ constants were used: 9, 60, 300 and 1000. Moreover, we suppose that the nodes are not homogenous, so these are randomly born with knowledge between 0 and 3 (little amount of information). Based on these assumptions, the analysis of the Knowledge Sharing based routing protocol attempts to quantify the knowledge properties of the Societies architecture. The analysis computes the dissemination of information, knowledge accumulated and learning frequencies.

\subsection{The Results}

Fig. 3 presents the number of nodes classified in each class. Class 1 contains the largest total number of nodes created. This is the class where nodes are initially born. Class 2 has fewer nodes classified than both classes 1 and even 3 . This is due to the 
fact that the threshold between class 1 and class 2 is relatively small (10 points of knowledge). Consequently, many nodes from class 2 do not spend too much time in this class and move rapidly to class 3 , sometimes not even going through it. On the other hand, the threshold between class $3\left(\alpha_{k}=100\right)$ and class $4\left(\alpha_{k}=1000\right)$ is larger, hence leading to a slower flow of societies between the two categories. Note that the choice of these disparate threshold values was made on purpose in order to highlight the evolution under such peculiar circumstances and highlights the importance of choosing $\alpha_{k}$.

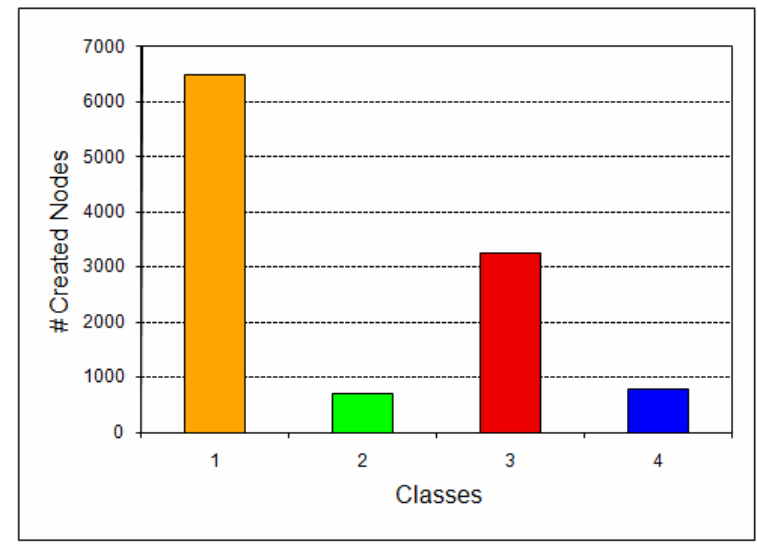

Fig. 3. Skype society

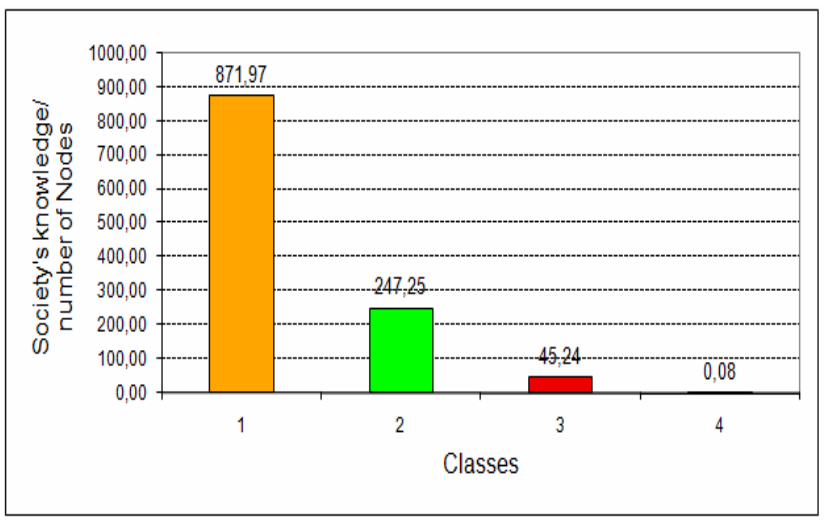

Fig. 4. Skype society

Fig 4. presents the total count of acquired knowledge per node of each class, so it also shows the evolution of nodes learning. Class 1 received more information, because a node from this class has a high probability to accept knowledge sharing. It follows, or mimics, the young behavior, because the learning allows the survival of 
nodes, and giving the necessary knowledge to cross over to a next class. This figure also presents the dissemination flow. We can see the knowledge flow; it decreases more when the node goes to a high class, as this has less information received.

Fig. 5 depicts the learning curve with the graphical relation between the average learning of nodes and the time taken to learn. Classes 2, 3 and 4 use the blue, red and green colors respectively. Due to limitations in the OMNET simulator, class 1 learning curve is not displayed due to the large number of values it has, more than the OMNET graphical tool may handle. It seems that knowledge stabilizes at some moment. The authors plan to conduct more simulations.

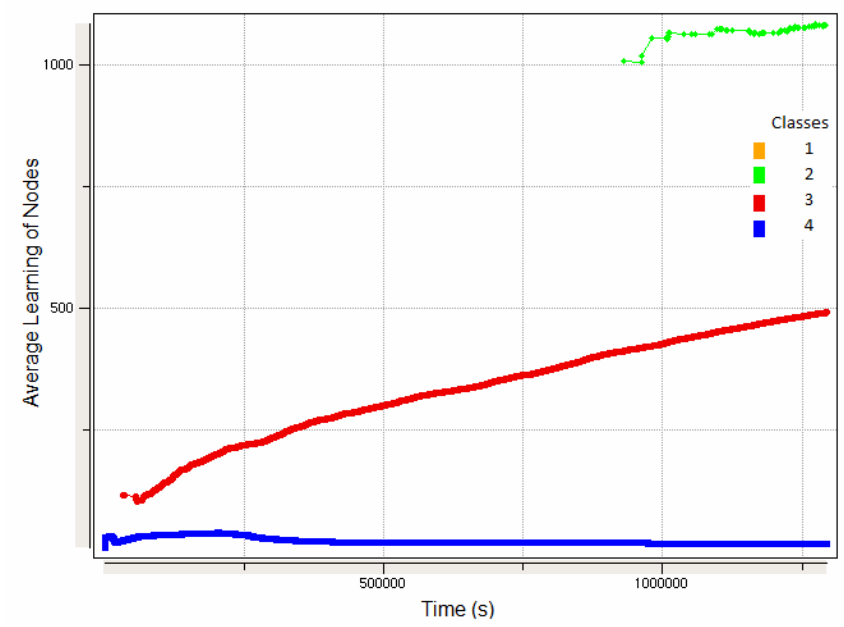

Fig. 5. Learning curve

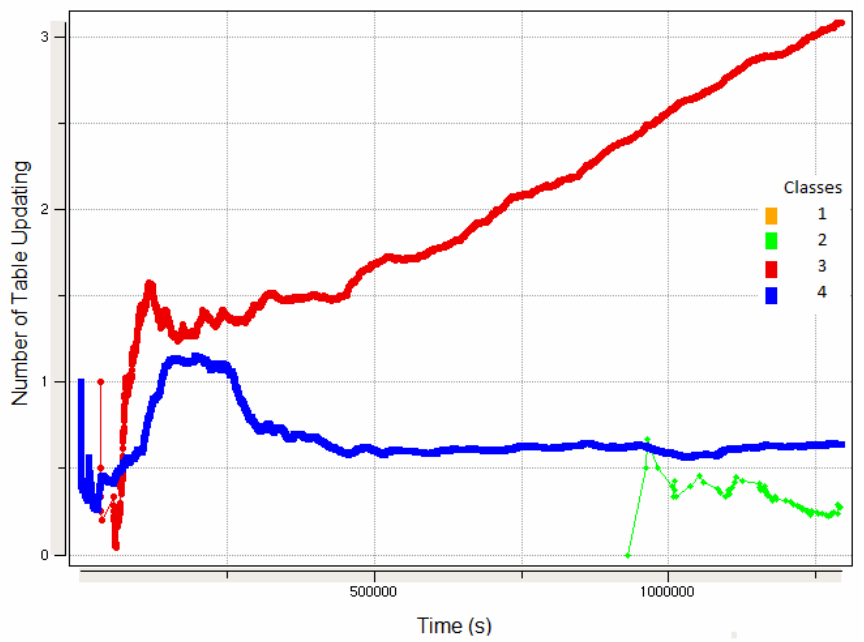

Fig. 6. Learning curve 
The knowledge sharing and routing algorithm was implemented using knowledge tables. Each node has a table that is updated when it receives information. Therefore, Fig. 6 shows the average number of table updating.

\section{Conclusions and Future Directions}

Routing is an important part of any future Internet architecture. Societies and their communication styles are expected to be at the core the architectural mechanisms. The author introduces an approach for quantifying the information sharing/routing among societies in Internet, based on the society classification and information adoption model, but we intend to analyze such model in-depth studies to understand inter-society information routing using real traces.

The initial experiment studied the evolution of societies and the information sharing among such simulated societies. Knowledge believe and probable forwarding based on trust, evidence have shown that despite leading to apparent unexpected non deterministic routing results, they could nonetheless be used as a basis for next Internet to reach all possible groups.

Problems such as email spam and unwanted traffic may be dealt with using such inter-society policy based routing. We plan to expand the simulations in order to gain more understanding of the results.

\section{References}

1. Smith, T.F., Waterman, M.S.: Identification of Common Molecular Subsequences. J. Mol. Biol. 147, 195-197 (1981)

2. Jacobson, V., Mosko, M., Smetters, D., Garcia-Luna-Aceves, J.J.: Content-Centric Networking. Whitepaper Describing Future Assurable Global Networks. Palo Alto Research Center, Inc. (January 30, 2007)

3. Baset, S.A., Schulzrinne, H.G.: An Analysis of the Skype Peer-to-Peer Internet Telephony Protocol. In: INFOCOM. 25th IEEE International Conference on Computer Communications, pp. 1-11 (2006)

4. Li, X.Y., Moaveninejad, K., Frieder, O.: Regional gossip routing for wireless ad hoc networks. Mobile Networks and Applications 10(1-2), 61-77 (2005)

5. Braginsky, D., Estrin, D.: Rumor Routing Algorithm For Sensor Networks. In: WSNA'02, USA (2002)

6. Dempster, A.P.: A generalization of Bayesian inference. Journal of the Royal Statistical Society, Series B 30, 205-247 (1968)

7. Shafer, G.: A Mathematical Theory of Evidence. Princeton University Press, Princeton (1976)

8. Cointet, J.-P., Roth, C.: How Realistic Should Knowledge Diffusion Models Be? Jour. of Artificial Societies and Social Simulation 10(3, 5) (2007)

9. Cowan, R.: Network structure and the diffusion of knowledge. Journal of Economic Dynamics and Control 28(8), 1557-1575 (2004)

10. Vahdat, A., Becker, D.: Epidemic routing for partially connected ad hoc networks, Duke University, Tech. Rep. CS-200006 (April 2000) 
11. Lindgren, A., Doria, A., Schelen, O.: Probabilistic Routing in Intermittently Connected Networks. In: 1st. International Workshop on Assurance with Partial and Intermittent Resources

12. Kenah, E., Robins, J.: Network-based analysis of stochastic SIR epidemic models with random and proportionate mixing. J. Theor. Biol. 249(4), 706 (2007)

13. Chen, X., Murphy, A.L.: Enabling disconnected transitive communication in mobile ad hoc networks. In: Proc. of Workshop on Principles of Mobile Computing, pp. 21-27 (2001)

14. Zhang, X., Neglia, G., Kurose, J., Towsley, D.: Performance Modeling of Epidemic Routing. MIT Rep. 05-44 (2005)

15. Hass, Z.J., Small, T.: A New Networking Model for Biological Applications of Ad Hoc Sensor Networks. IEEE/ACM Transactions on Networking (February 2006)

16. Spyropoulos, T., Psounis, K., Raghavendra, C.: Efficient Routing in Intermittently Connected Mobile Networks: The Multi-copy Case. ACM/IEEE Journal of Transactions on Networking (2007)

17. Lipsa, G.: Routing Strategy for Minimizing the Packet Loss in Disruptive Tolerant Networks. Information Sciences and Systems, 1167-1172 (2008)

18. Sadok, D.H., França, J., Abreu, R., Oliveira, L.: Knowledge Sharing to Improve Routing and Future 4G Networks. In: Adibi, S., Mobasher, A., Tofighbakhsh, M. (eds.) FourthGeneration Wireless Networks: Applications and Innovations IGI-Global, ch. 10 (2009) ISBN: 9781615206742

19. Han, S., et al.: Collaborative Blog Spam Filtering Using Adaptive Percolation Search. In: WWW 2006, Edinburgh, UK, May 22-26 (2006)

20. Moore, C., Newman, M.: Epidemics and Percolation in Small-world Networks. Physical Review 61(5), 5678-5682 (2001) 